\title{
APPLICATION OF CHIRAL RESONANCE LAGRANGIAN THEORIES TO THE MUON $g-2^{*}$
}

\author{
FRED JEGERLEHNER \\ Institute of Physics, Humboldt-Universität zu Berlin \\ Newtonstr. 15, 12489 Berlin, Germany \\ and \\ DESY, Platanenallee 6, 15738 Zeuthen, Germany
}

(Received October 24, 2013)

We think that phenomenological resonance Lagrangian models, constrained by global fits from the low energy hadron reaction data, can help to improve muon $g-2$ predictions. The main issue are those contributions which cannot be calculated by perturbative means: the hadronic vacuum polarization (HVP) effects and the hadronic light-by-light (HLbL) scattering contribution. I review the recent progress in the evaluation of the HVP contribution within the broken Hidden Local Symmetry (HLS) framework, worked out in collaboration with Benayoun, David and DelBuono. Our HLS driven estimate reads $a_{\mu}^{\mathrm{LO} \text { had }}=(688.60 \pm 4.24) \times 10^{-10}$ and we find $a_{\mu}^{\text {the }}=(11659177.65 \pm 5.76) \times 10^{-10}$.

DOI:10.5506/APhysPolB.44.2257

PACS numbers: 14.60.Ef, 13.40.Em

\section{Effective field theory: the Resonance Lagrangian Approach}

The Resonance Lagrangian Approach (RLA) provides an extension of low energy effective QCD as represented by the Chiral Perturbation Theory (ChPT) to energies up to about $1 \mathrm{GeV}$. Principles to be included are the chiral structure of QCD, the vector-meson dominance model and electromagnetic gauge invariance. Specifically, we will consider the HLS version, which is considered to be equivalent to alternative variants after implementing the appropriate high energy asymptotic conditions. ChPT is the systematic and unambiguous approach to the low energy effective QCD given by spontaneously broken chiral symmetry $\mathrm{SU}(3) \otimes \mathrm{SU}(3)$, with the pseudoscalars as

* Presented at the XXXVII International Conference of Theoretical Physics "Matter to the Deepest" Ustron, Poland, September 1-6, 2013. 
Nambu-Goldstone bosons, together with a systematic expansion in low momenta and chiral symmetry breaking (SB) effects by the light quark masses, $m_{q}, q=u, d, s$. The limitation of ChPT is the fact that it ceases to converge for energies above about $400 \mathrm{MeV}$, in particular it lacks to describe physics involving the vector resonances $\rho, \omega$ and $\phi$.

The Vector-meson Dominance Model (VDM) is the effective theory implementing the direct coupling of the neutral spin 1 vector resonances $\rho, \omega, \phi$ etc. to the photon. Such direct couplings are a consequence of the fact that the neutral spin 1 resonances like the $\rho^{0}$ are composed of charged quarks. The effect is well modeled by the VDM Lagrangian $\mathcal{L}_{\gamma \rho}=\frac{e}{2 g_{\rho}} \rho_{\mu \nu} F^{\mu \nu}$ or $=-\frac{e M_{\rho}^{2}}{g_{\rho}} \rho_{\mu} A^{\mu}$, which has to be implement in the low energy effective QCD in a way which is consistent with the chiral structure of QCD.

The construction of the HLS model may be outlined as follows: like in ChPT the basic fields are the unitary matrix fields $\xi_{\mathrm{L}, \mathrm{R}}=\exp \left[ \pm \mathrm{i} P / f_{\pi}\right]$, where $P=P_{8}+P_{0}$ is the $\mathrm{SU}(3)$ matrix of pseudoscalar fields, with $P_{0}$ and $P_{8}$ the basic singlet and octet fields, respectively. The pseudoscalar field matrix $P$ is represented by

$$
\begin{aligned}
& P_{8}=\frac{1}{\sqrt{2}}\left(\begin{array}{ccc}
\frac{1}{\sqrt{2}} \pi_{3}+\frac{1}{\sqrt{6}} \eta_{8} & \pi^{+} & K^{+} \\
\pi^{-} & -\frac{1}{\sqrt{2}} \pi_{3}+\frac{1}{\sqrt{6}} \eta_{8} & K^{0} \\
K^{-} & \bar{K}^{0} & -\sqrt{\frac{2}{3}} \eta_{8}
\end{array}\right), \\
& P_{0}=\frac{1}{\sqrt{6}} \operatorname{diag}\left(\eta^{0}, \eta^{0}, \eta^{0}\right) ; \quad\left(\pi_{3}, \eta_{8}, \eta_{0}\right) \Leftrightarrow\left(\pi^{0}, \eta, \eta^{\prime}\right) .
\end{aligned}
$$

The HLS ansatz is an extension of the ChPT non-linear sigma model to a non-linear chiral Lagrangian $\left[\operatorname{Tr} \partial_{\mu} \xi^{+} \partial^{\mu} \xi\right]$ based on the symmetry pattern $G_{\text {global }} / H_{\text {local }}$, where $G=\mathrm{SU}(3)_{\mathrm{L}} \otimes \mathrm{SU}(3)_{\mathrm{R}}$ is the chiral group of QCD and $H=\mathrm{SU}(3)_{\mathrm{V}}$ the vector subgroup. The hidden local $\mathrm{SU}(3)_{\mathrm{V}}$ requires the spin 1 vector meson fields, represented by the $\mathrm{SU}(3)$ matrix field $V_{\mu}$, to be gauge fields. The needed covariant derivative reads $D_{\mu}=\partial_{\mu}-i g V_{\mu}$, and allows to include the couplings to the electroweak gauge fields $A_{\mu}, Z_{\mu}$ and $W_{\mu}^{ \pm}$in a natural way. The vector field matrix is usually written as

$$
V=\frac{1}{\sqrt{2}}\left(\begin{array}{ccc}
\left(\rho^{I}+\omega^{I}\right) / \sqrt{2} & \rho^{+} & K^{*+} \\
\rho^{-} & \left(-\rho^{I}+\omega^{I}\right) / \sqrt{2} & K^{* 0} \\
K^{*-} & \bar{K}^{* 0} & \phi^{I}
\end{array}\right) .
$$


The unbroken HLS Lagrangian is then given by

$$
\mathcal{L}_{\mathrm{HLS}}=\mathcal{L}_{\mathrm{A}}+\mathcal{L}_{\mathrm{V}} ; \quad \mathcal{L}_{\mathrm{A} / \mathrm{V}}=-\frac{f_{\pi}^{2}}{4} \operatorname{Tr}[L \pm R]^{2},
$$

where $L=\left[D_{\mu} \xi_{\mathrm{L}}\right] \xi_{\mathrm{L}}^{+}$and $R=\left[D_{\mu} \xi_{\mathrm{R}}\right] \xi_{\mathrm{R}}^{+}$. The covariant derivatives read

$$
\left\{\begin{array}{c}
D_{\mu} \xi_{\mathrm{L}}=\partial_{\mu} \xi_{\mathrm{L}}-i g V_{\mu} \xi_{\mathrm{L}}+i \xi_{\mathrm{L}} \mathcal{L}_{\mu} \\
D_{\mu} \xi_{\mathrm{R}}=\partial_{\mu} \xi_{\mathrm{R}}-i g V_{\mu} \xi_{\mathrm{R}}+i \xi_{\mathrm{R}} \mathcal{R}_{\mu}
\end{array}\right.
$$

with known couplings to the Standard Model (SM) gauge bosons

$$
\left\{\begin{array}{l}
\mathcal{L}_{\mu}=e Q A_{\mu}+\frac{g_{2}}{\cos \theta_{W}}\left(T_{z}-\sin ^{2} \theta_{W}\right) Z_{\mu}+\frac{g_{2}}{\sqrt{2}}\left(W_{\mu}^{+} T_{+}+W_{\mu}^{-} T_{-}\right) \\
\mathcal{R}_{\mu}=e Q A_{\mu}-\frac{g_{2}}{\cos \theta_{W}} \sin ^{2} \theta_{W} Z_{\mu}
\end{array} .\right.
$$

Like in the electroweak SM, masses of the spin 1 bosons may be generated by the Higgs-Kibble mechanism if one starts in place of the non-linear $\sigma$-model with the Gell-Mann-Levy linear $\sigma$-model by a shift of the $\sigma$-field.

In fact, the global chiral symmetry $G_{\text {global }}$ is well known not to be realized as an exact symmetry in nature, which implies that the ideal HLS symmetry evidently is not a symmetry of nature either. It evidently has to be broken appropriately in order to provide a realistic low energy effective theory mimicking low energy effective QCD. Corresponding to the strength of the breaking, usually, this is done in two steps, breaking of $\mathrm{SU}(3)$ in a first step and breaking the isospin $\mathrm{SU}(2)$ subgroup in a second step. Unlike in ChPT (perturbed non-linear $\sigma$-model) where one is performing a systematic low energy expansion, expanding in low momenta and the quark masses, here we introduce symmetry breaking as phenomenological parameters to be fixed from appropriate data, since a systematic low energy expansion à la ChPT ceases to converge at energies above about $400 \mathrm{MeV}$, while we attempt to model phenomenology up to including the $\phi$ resonance.

The broken HLS Lagrangian (BHLS) is then given by (see [1])

$$
\mathcal{L}_{\mathrm{BHLS}}=\mathcal{L}_{\mathrm{A}}^{\prime}+\mathcal{L}_{\mathrm{V}}^{\prime}+\mathcal{L}_{\text {'t Hooft }} ; \mathcal{L}_{\mathrm{A} / \mathrm{V}}^{\prime}=-\frac{f_{\pi}^{2}}{4} \operatorname{Tr}\left\{[L \pm R] X_{\mathrm{A} / \mathrm{V}}\right\}^{2},
$$

with 6 phenomenological chiral SB parameters. The phenomenological SB pattern suggests $X_{\mathrm{I}}=\operatorname{diag}\left(q_{\mathrm{I}}, y_{\mathrm{I}}, z_{\mathrm{I}}\right),\left|q_{\mathrm{I}}-1\right|,\left|y_{\mathrm{I}}-1\right| \ll\left|z_{\mathrm{I}}-1\right|, \mathrm{I}=\mathrm{V}, \mathrm{A}$. There is also the parity odd anomalous sector, which is needed to account for reactions like $\gamma^{*} \rightarrow \pi^{0} \gamma$ and $\gamma^{*} \rightarrow \pi^{+} \pi^{-} \pi^{0}$ among others. 
We note that this BHLS model would be a reliable low energy effective theory if the QCD scale $\Lambda_{\mathrm{QCD}}$ would be large relative to the scale of about $1 \mathrm{GeV}$ up to which we want to apply the model, which in reality is not the case. Nevertheless, as a phenomenological model applied to low multiplicity hadronic processes (specified below) it seems to work pretty well, as we have demonstrated by a global fit of the available data in Ref. [1]. The major achievement is a simultaneous consistent fit of the $e^{+} e^{-} \rightarrow \pi^{+} \pi^{-}$data from CMD-2 [2], SND [3], KLOE [4] and BaBar [5], and the $\tau \rightarrow \pi^{-} \pi^{0} \nu_{\tau}$ decay spectral functions by ALEPH [6], OPAL [7], CLEO [8] and Belle [9]. The $e^{+} e^{-} \rightarrow \pi^{-} \pi^{+}$channel gives the dominant hadronic contribution to the muon $g-2$. Isospin symmetry $\pi^{-} \pi^{0} \Leftrightarrow \pi^{-} \pi^{+}$allows one to include existing high quality $\tau$-data as advocated long time ago in [10].

We note that as long as higher order corrections are restricted to the mandatory pion- and kaon-loop effects in the vector boson self-energies, renormalizability is not an issue. These contributions behave as in a strictly renormalizable theory and correspond to a reparametrization only.

\section{2. $\rho^{0}-\gamma$ mixing solving the $\tau$ versus $e^{+} e^{-}$puzzle}

A minimal subset of any resonance Lagrangian is given by the VDM + scalar QED part which describes the leading interaction between the $\rho$ the pions and the photon. In order to account for the decay of the $\rho$, one has to include self-energy effects, which also affect $\rho-\gamma$ mixing via pion-loops shown in Fig. 1. Most previous calculations, considered the mixing term to be a constant, and were missing a substantial quantum interference effect.

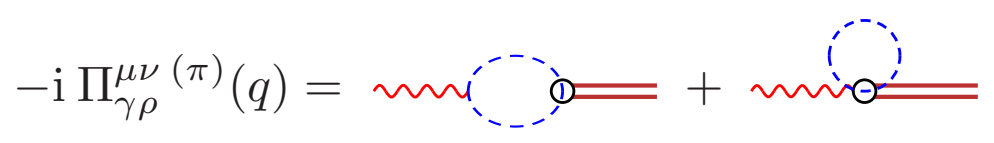

Fig. 1. Irreducible self-energy contribution at one-loop.

The properly normalized pion form factor, in our approach, has the from

$$
F_{\pi}(s)=\left[e^{2} D_{\gamma \gamma}+e\left(g_{\rho \pi \pi}-g_{\rho e e}\right) D_{\gamma \rho}-g_{\rho e e} g_{\rho \pi \pi} D_{\rho \rho}\right] /\left[e^{2} D_{\gamma \gamma}\right],
$$

with propagators including the pion loop effects, with typical couplings $g_{\rho \pi \pi \text { bare }}=5.8935, g_{\rho \pi \pi \text { ren }}=6.1559, g_{\rho e e}=0.018149, x=g_{\rho \pi \pi} / g_{\rho}=$ 1.15128 , fixed from the (partial) widths

$$
g_{\rho \pi \pi}=\sqrt{48 \pi \Gamma_{\rho} /\left(\beta_{\rho}^{3} M_{\rho}\right)} ; \quad g_{\rho e e}=\sqrt{12 \pi \Gamma_{\rho e e} / M_{\rho}} .
$$


The effect of taking into account or not the $\gamma-\rho^{0}$ mixing is illustrated in Fig. 2. The $\gamma-\rho$ interference is crucial when relating charged current $\tau$-data to $e^{+} e^{-}$-data. Including known isospin breaking (IB) corrections $v_{0}(s)=R_{\mathrm{IB}}(s) v_{-}(s)$, a large discrepancy [ 10\%] persisted [11], which was known as the $\tau$ versus $e^{+} e^{-}$puzzle since [12]. In [13], it has been shown that the $\gamma-\rho$ mixing active in the $e^{+} e^{-} \rightarrow \pi^{+} \pi^{-}$channel is responsible for the discrepancy, i.e. $\tau$-data have to be corrected as $v_{0}(s)=r_{\rho \gamma}(s) R_{\mathrm{IB}}(s) v_{-}(s)$, before they can be used as representing an equivalent $I=1 e^{+} e^{-} \rightarrow \pi^{+} \pi^{-}$ data sample (see also $[14,15])$. Note that what goes into $a_{\mu}$ directly are the $e^{+} e^{-}$-data. Best "proof" of the required $\rho-\gamma$ correction profile is the ALEPH versus BaBar fit shown in Fig. 1 of [16]. Applying the correction to the $\tau$-spectra (see Fig. 8 in [13]) implies a universal shift down by $\delta a_{\mu}^{\text {had }}[\rho \gamma] \simeq(-5.1 \pm 0.5) \times 10^{-10}$ of the contribution to the muon $g-2$. This shift brings into agreement the $\tau$ inclusive estimates with the $e^{+} e^{-}$based ones. Is our model, treating pions as point-like objects, viable? A good "answer" to this question may be obtained by looking at the $\pi \pi$ production in $\gamma \gamma$ fusion. Figure 3 shows: at the strong tensor meson resonance $f_{2}(1270)$ in the $\pi \pi$ channel, photons directly probe the quarks! However, in the region of our interest, photons see pions (below about $1 \mathrm{GeV}$ ). We apply the sQED model up to $0.975 \mathrm{GeV}$ (relevant for $a_{\mu}$ ), which should be rather reliable. Switching off the electromagnetic interaction of pions, is definitely not a realistic approximation in trying to describe what is observed in the $e^{+} e^{-} \rightarrow \pi^{+} \pi^{-}$channel.

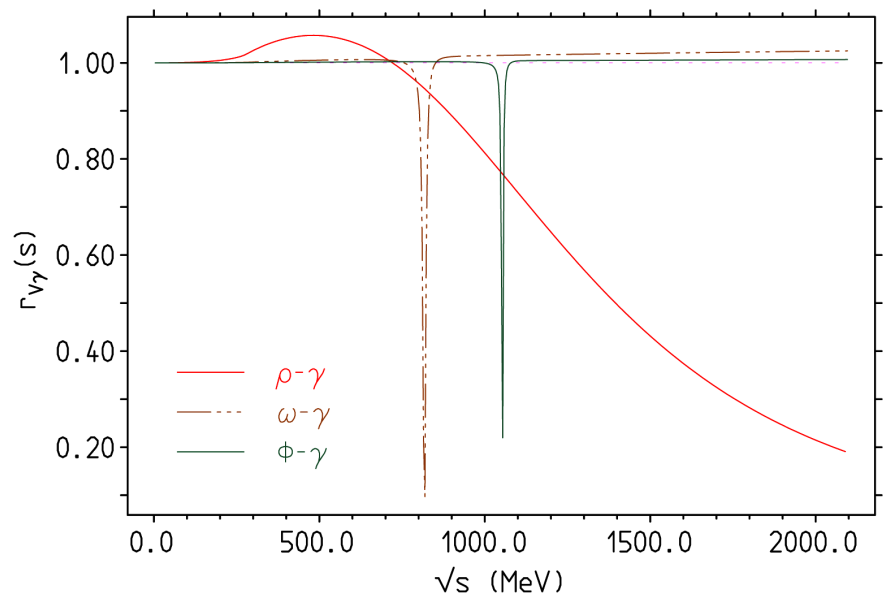

Fig. 2. Ratio of the full $\left|F_{\pi}(s)\right|^{2}$ in units of the same quantity omitting the mixing term (full line). Also shown is the same mechanism scaled up by the branching fraction $\Gamma_{\mathrm{V}} / \Gamma(V \rightarrow \pi \pi)$ for $V=\omega$ and $\phi$. In the $\pi \pi$ channel, the effects for resonances $V \neq \rho$ are tiny if not very close to resonance. 


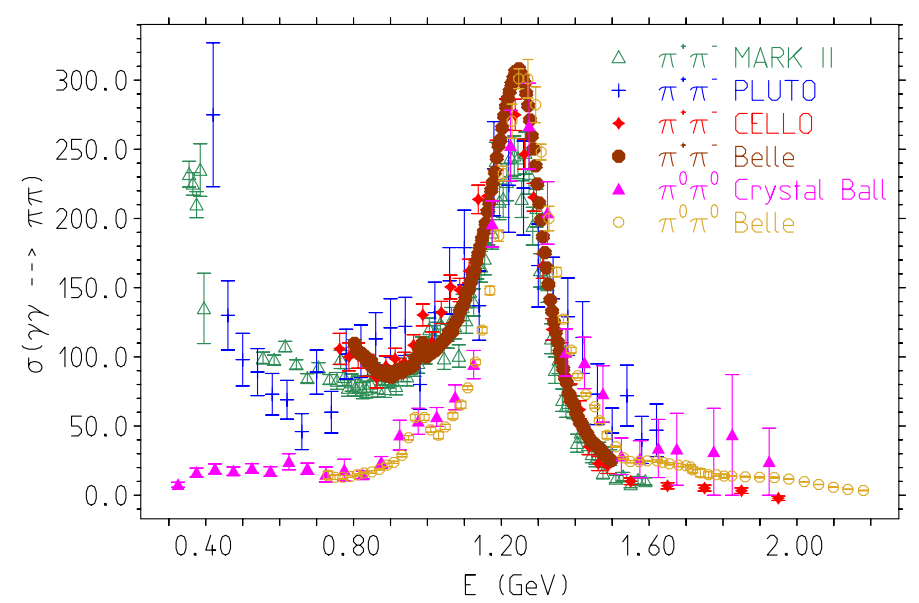

Fig. 3. How photons couple to pions? This is obviously probed in reactions like $\gamma \gamma \rightarrow \pi^{+} \pi^{-}, \pi^{0} \pi^{0}$. Data infer that below about $1 \mathrm{GeV}$ photons couple to pions as point-like objects (i.e. to the charged ones overwhelmingly). At higher energies, the photons see the quarks exclusively and form the prominent tensor resonance $f_{2}(1270)$. The $\pi^{0} \pi^{0}$ cross section shown has been multiplied by the isospin symmetry factor 2 , by which it is reduced in reality.

\section{Global fit of BHLS parameters and prediction of $\boldsymbol{F}_{\pi}(s)$}

The simple model just considered illustrates one of the main quantum interference effects in the isospin sector, the $\gamma-\rho^{0}$ mixing. A more complete effective theory must include the $\rho^{0}-\omega$ mixing, as well as the strangeness sector, with the kaons as additional pseudo Nambu-Goldstone bosons, including the $\eta$ and the $\eta^{\prime}$, and the mixing with the $\phi$. This is implemented in the BHLS model introduced before. Self-energy corrections for $\rho, \omega, \phi$ and $\gamma$ now include kaon-loops as well. In addition, parity odd sector contributions like $\pi^{0} \rightarrow \gamma \gamma$ and $\gamma \rightarrow \pi^{+} \pi^{-} \pi^{0}$ must be included. At present there are 45 different data sets ( 6 annihilation channels and 10 partial width decays) available below $E_{0}=1.05 \mathrm{GeV}$ (just above the $\phi$ ), and we use them to constrain the BHLS Lagrangian couplings. The method is able to reduce uncertainties in $g-2$ predictions by using indirect constraints on the Lagrangian parameters.

The main goal is to single out a representative effective resonance Lagrangian by the global fit. The constrained model is expected to help in improving model calculations of hadronic light-by-light scattering. The new muon $g-2$ experiments planned at Fermilab and J-PARC, supposed to start in about 2-3 years, are expected to reduce experimental errors by a factor 4 . On the theory side, this requires a comparable improvement of the HVP and HLbL contributions. 
The effective theory predicts the cross sections $\pi^{+} \pi^{-}, \pi^{0} \gamma, \eta \gamma, \eta^{\prime} \gamma$, $\pi^{0} \pi^{+} \pi^{-}, K^{+} K^{-}, K^{0} \bar{K}^{0}$ which account $83.4 \%$ of the HVP contribution to the muon $g-2$. Contributions from the missing channels $4 \pi, 5 \pi, 6 \pi, \eta \pi \pi, \omega \pi$ and from higher energies we evaluate using data directly and pQCD in the perturbative region and in the tail. The resulting BHLS prediction for $a_{\mu}^{\mathrm{LO} \text {,had }}$ allows us to get a BHLS driven SM prediction for $a_{\mu}$ (see Table I).

TABLE I

Standard Model theory and experiment comparison [in units $10^{-11}$ ].

\begin{tabular}{|c|c|c|}
\hline Contribution & Value & Error \\
\hline QED incl. 4-loops +5 -loops & 116584718.85 & 0.04 \\
\hline Leading hadronic vacuum polarization & 6886.0 & 42.4 \\
\hline Subleading hadronic vacuum polarization & -98.32 & 0.82 \\
\hline Hadronic light-by-light & 116.0 & 39.0 \\
\hline Weak incl. 2-loops & 154.0 & 1.0 \\
\hline Theory & 116591776.5 & 57.6 \\
\hline Experiment & 116592089.0 & 63.0 \\
\hline Exp.-The. 3.7 standard deviations & 312.5 & 85.4 \\
\hline
\end{tabular}

Our favored evaluation based on selected data yields $a_{\mu}^{\mathrm{LO}}$ had $=(681.23 \pm$ $4.51) \times 10^{-10}$ and a prediction $a_{\mu}^{\text {the }}=(11659170.28 \pm 5.96) \times 10^{-10}$ and $\Delta a_{\mu}=$ $a_{\mu}^{\exp }-a_{\mu}^{\text {the }}=\left(38.52 \pm 5.96_{\text {the }} \pm 6.3_{\exp }\right) \times 10^{-10}$. The associated fit probability is $94 \%$ and the significance for $\Delta a_{\mu}$ is $4.4 \sigma$. Including all data, applying appropriate rewighting in the case of inconsistencies ${ }^{1}$, we find $a_{\mu}^{\mathrm{LO} \text { had }}=$ $(688.60 \pm 4.24) \times 10^{-10}$ such that $a_{\mu}^{\text {the }}=(11659177.65 \pm 5.76) \times 10^{-10}$ and $\Delta a_{\mu}=a_{\mu}^{\exp }-a_{\mu}^{\text {the }}=\left(31.25 \pm 5.76_{\text {the }} \pm 6.3_{\exp }\right) \times 10^{-10}$. The associated fit probability is $76 \%$ and the significance for $\Delta a_{\mu}$ is $3.7 \sigma$. The comparison of our global fit result with other results from DHMZ10 [16, 19], JS11 [13], DHea09 [11], HLMNT11 [20] is shown in Fig. 4. We get somewhat lower central values than results obtained by direct integration of the data, but all results agree well within $1 \sigma$. Our fits, which include the $\tau$-data, exhibit the best fit probability for KLOE10 results, while there is some tension showing up in case of the BaBar $\pi \pi$ data. Our analysis has been criticized lately in Ref. [21] but what is shown in that reply is that BaBar [5] and KLOE data are not quite compatible within the given experimental errors. A different issue is the comparison between BaBar and $\tau$ spectral data. Contrary to claims in [21], the sizable $\gamma-\rho^{0}$ mixing effect has not been taken into account and

\footnotetext{
${ }^{1}$ The required rewighting concerns the $e^{+} e^{-} \rightarrow \pi^{+} \pi^{-} \pi^{0}$ data in the vicinity of the $\phi$, as well as the KLOE08 and the BaBar $e^{+} e^{-} \rightarrow \pi^{+} \pi^{-}$data sets.
} 
one should see a substantial shift which, however, is found to be absent in the comparison between Belle $\tau$-data and the BaBar $e^{+} e^{-}$data (see Fig. 1 in [16]).

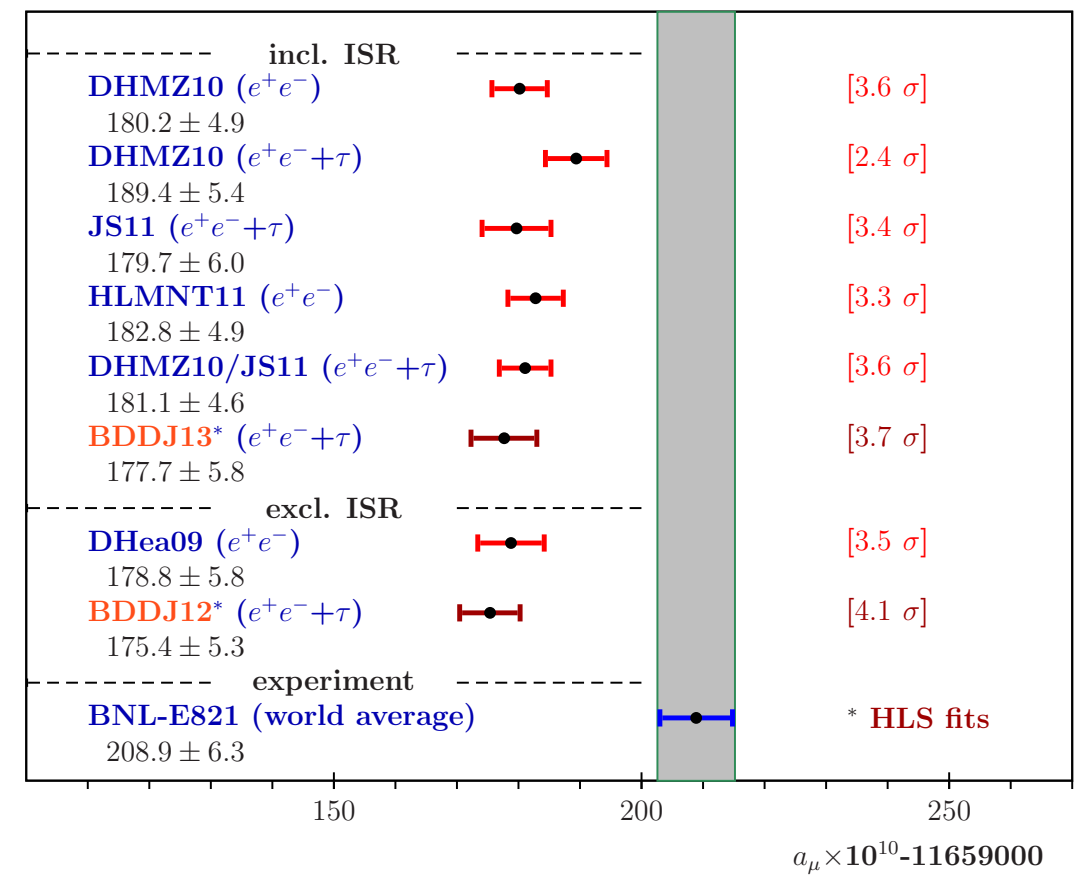

Fig. 4. Comparison with other results. Note: results depend on which value is taken for HLbL. JS11 and BDDJ13 includes 116(39) $\times 10^{-11}$ (JN [17]), DHea09, DHMZ10, HLMNT11 and BDDJ12 use 105(26) $\times 10^{-11}(\mathrm{PdRV}[18])$.

A comparison between theory and experiment [22] is given in Table I (see also [23]). Theory results shown are updates from Ref. [17] using results on improved 4-loop and the new 5-loop QED corrections [24], improved lepton mass ratios [25] and using the new Higgs mass value from ATLAS and CMS in the evaluation of the weak corrections [26].

\section{Lessons and outlook}

Effective field theory is the only way to understand relationships between different channels, like $e^{+} e^{-}$-annihilation cross-sections and $\tau$-decay spectra. Global fit strategies allow to single out variants of the effective resonance Lagrangian models. Models for individual channels can parametrize data, but do not allow to understand them and their relation to other channels. We get perfect fits for $\left|F_{\pi}(s)\right|^{2}$ up to just above the $\phi$ without higher 
$\rho^{\prime}$ 's $\rho^{\prime}, \rho^{\prime \prime}$, which seem to be mandatory in the Gounaris-Sakurai type fits. $\tau$-data in our approach play a special role, because they are much simpler than the $e^{+} e^{-}$data, which exhibit intricate $\gamma-\rho^{0}-\omega-\phi$ mixing effects.

RLA type analyses provide analytic shapes for amplitudes, and such "physical shape information" is favorable over ad hoc data interpolations, the simplest being the trapezoidal rule, which is known to be problematic when data are sparse or strongly energy dependent.

Limitations of the RLA are the large couplings which make systematic higher order improved analyses problematic. As illustrated by Fig. 3, considering pions and kaons to be point-like may be not too bad an approximation, in the range we are applying the model. Also, we consider our analysis as a starting point to be confronted with other RLA versions and implementations and with what happens if one tries to include higher order effects.

Many thanks to the Organizers for the invitation and support to the 2013 "Matter to the Deepest" International Conference at Ustron, Poland, and for giving me the opportunity to present this talk.

\section{REFERENCES}

[1] M. Benayoun, P. David, L. DelBuono, F. Jegerlehner, Eur. Phys. J. C72, 1848 (2012); Eur. Phys. J. C73, 2453 (2013).

[2] R.R. Akhmetshin et al. [CMD-2 Collaboration], Phys. Lett. B578, 285 (2004); Phys. Lett. B648, 28 (2007).

[3] M.N. Achasov et al., J. Exp. Theor. Phys. 103, 380 (2006) [Zh. Eksp. Teor. Fiz. 130, 437 (2006)].

[4] F. Ambrosino et al. [KLOE Collaboration], Phys. Lett. B670, 285 (2009); Phys. Lett. B700, 102 (2011); D. Babusci et al. [KLOE Collaboration], Phys. Lett. B720, 336 (2013).

[5] B. Aubert et al. [BaBar Collaboration], Phys. Rev. Lett. 103, 231801 (2009); J.P. Lees et al. [BaBar Collaboration], Phys. Rev. D86, 032013 (2012).

[6] S. Schael et al. [ALEPH Collaboration], Phys. Rep. 421, 191 (2005).

[7] K. Ackerstaff et al. [OPAL Collaboration], Eur. Phys. J. C7, 571 (1999).

[8] S. Anderson et al. [CLEO Collaboration], Phys. Rev. D61, 112002 (2000).

[9] M. Fujikawa et al. [Belle Collaboration], Phys. Rev. D78, 072006 (2008).

[10] R. Alemany, M. Davier, A. Höcker, Eur. Phys. J. C2, 123 (1998).

[11] M. Davier et al., Eur. Phys. J. C66, 127 (2010).

[12] M. Davier, S. Eidelman, A. Höcker, Z. Zhang, Eur. Phys. J. C27, 497 (2003).

[13] F. Jegerlehner, R. Szafron, Eur. Phys. J. C71, 1632 (2011). 
[14] M. Benayoun et al., Eur. Phys. J. C55, 199 (2008).

[15] M. Benayoun, P. David, L. DelBuono, O. Leitner, Eur. Phys. J. C65, 211 (2010); Eur. Phys. J. C68, 355 (2010).

[16] M. Davier et al., Eur. Phys. J. C66, 1 (2010).

[17] F. Jegerlehner, A. Nyffeler, Phys. Rep. 477, 1 (2009); F. Jegerlehner, Springer Tracts Mod. Phys. 226, 1 (2008); Acta Phys. Pol. B 40, 3097 (2009).

[18] J. Prades, E. de Rafael, A. Vainshtein, arXiv:0901.0306 [hep-ph].

[19] M. Davier, A. Höcker, B. Malaescu, Z. Zhang, Eur. Phys. J. C71, 1515 (2011) [Erratum ibid. C72, 1874 (2012)].

[20] K. Hagiwara et al., J. Phys. G 38, 085003 (2011).

[21] M. Davier, B. Malaescu, arXiv:1306.6374 [hep-ex].

[22] G.W. Bennett et al. [Muon G-2 Collaboration], Phys. Rev. D73, 072003 (2006).

[23] J.P. Miller, E. d. Rafael, B.L. Roberts, D. Stöckinger, Ann. Rev. Nucl. Part. Sci. 62, 237 (2012).

[24] T. Aoyama, M. Hayakawa, T. Kinoshita, M. Nio, Phys. Rev. Lett. 109, 111808 (2012).

[25] P.J. Mohr, B.N. Taylor, D.B. Newell, Rev. Mod. Phys. 84, 1527 (2012).

[26] C. Gnendiger, D. Stöckinger, H. Stöckinger-Kim, Phys. Rev. D88, 053005 (2013). 